\title{
The Bursting Pulsar GRO J1744-28: the Slowest Transitional Pulsar?
}

\author{
J.M.C. Court ${ }^{1}$, D. Altamirano ${ }^{1} \&$ A. Sanna ${ }^{2}$ \\ ${ }^{1}$ Department of Physics and Astronomy, University of Southampton, Southampton, SO17 1BJ, UK \\ ${ }^{2}$ Dipartimento di Fisica, Università degli Studi di Cagliari, SP Monserrato-Sestu km 0.7, 09042 Monserrato, Italy
}

18 April 2018

\begin{abstract}
GRO J1744-28 (the Bursting Pulsar) is a neutron star LMXB which shows highly structured X-ray variability near the end of its X-ray outbursts. In this letter we show that this variability is analogous to that seen in Transitional Millisecond Pulsars such as PSR J1023+0038: 'missing link' systems consisting of a pulsar nearing the end of its recycling phase. As such, we show that the Bursting Pulsar may also be associated with this class of objects. We discuss the implications of this scenario; in particular, we discuss the fact that the Bursting Pulsar has a significantly higher spin period and magnetic field than any other known Transitional Pulsar. If the Bursting Pulsar is indeed transitional, then this source opens a new window of oppurtunity to test our understanding of these systems in an entirely unexplored physical regime.
\end{abstract}

Key words: stars: pulsars - accretion discs - instabilities - X-rays: binaries - X-rays: individual: GRO J1744-28

\section{INTRODUCTION}

Millisecond Pulsars are old radio pulsars with spin periods of order $\sim 10 \mathrm{~ms}$ (Backer et al. 1982). They have long been believed to be the end product of systems containing a neutron star (NS) in a Low Mass X-ray Binary (LMXB). In these systems, matter from a Roche-lobe overflowing star donates angular momentum to a NS, spinning it up to frequencies of several $100 \mathrm{~Hz}$ (Alpar et al. 1982). A number of fast-spinning X-ray pulsars (accreting Millisecond Pulsars, or AMXPs) have been found in LMXBs (e.g. Wijnands \& van der Klis 1998; Altamirano et al. 2008; Patruno et al. 2017; Sanna et al. 2017a), seemingly confirming this physical picture. At the end of this so-called 'recycling' process, the system should transition from an accretion-powered pulsar to a rotation-powered pulsar. As such, it has long been expected that such a transition could be observed by finding a system which changes its character from an accreting NS at one time to a radio pulsar at some later time. Subsequently a small family of 7 candidate objects have been discovered or proposed: these are referred to as Transitional Millisecond Pulsars (TMSPs).

The first of these objects, PSR J1023+0038, was identified by Archibald et al. 2009. Although it appeared as a non-accreting radio pulsar at the time of identification in 2009 , previous optical studies showed that this system contained an accretion disk in 2002 (Szkody et al. 2003). As such, the pulsar in this system must have switched from an accreting phase to a radio pulsar phase at some point be- tween 2003 and 2009, strongly suggesting the identification of this system as a TMSP. The pulsar in this system has a spin period of $1.69 \mathrm{~ms}$, and the companion is a star with a mass between $\sim 0.14-0.42 \mathrm{M}_{\odot}$. Archibald et al. 2009 suggested that the low X-ray luminosity of PSR J1023+0038 in its accreting phase was due to accretion taking place in the 'propeller regime' (Illarionov \& Sunyaev 1975). In this regime, accreting matter is halted by magnetic pressure above the co-rotation radius of the NS magnetosphere. This matter is then ejected from the system as a wind. Whether a system is in the propeller regime depends on its spin and its magnetic field strength (Lewin et al. 1988). Additionally, below a certain accretion rate, no stable balance between ram pressure and radiation pressure can form and any disk is ejected from the system (e.g. Campana et al. 1998). Archibald et al. 2009 suggested that the current accretion rate in PSR J1023+0038 is only slightly below this critical value, and that any small increase in accretion rate could cause accretion in this system to resume. They suggested the possibility of TMSP systems which flip back and forth between accreting and radio pulsar phases multiple times.

Papitto et al. 2013a identified IGR J18245-2452 as the first pulsar to switch from a radio pulsar to an AMXP and back to a radio pulsar. This source was first observed as a radio pulsar (Manchester et al. 2005), before being observed several years later by XMM-Newton (Eckert et al. 2013) as an AMXP. Several months after the XMM-Newton 
observation, Papitto et al. 2013b found that the source had reactivated as a radio pulsar during $\mathrm{X}$-ray quiescence. The pulsar in this system has a period of $3.93 \mathrm{~ms}$, and the companion star has a mass of $>0.17 \mathrm{M}_{\odot}$ (Papitto et al. 2013a). During the 2013 outburst of IGR J18245-2452, Ferrigno et al. 2014 reported the presence of high-amplitude variability in the X-ray lightcurve. They interpreted this as being due to the accretion rate $\dot{M}$ being very close to the critical rate at which the propeller effect begins to dominate the flow geometry. In this regime, small fluctuations in $\dot{M}$ cause so-called 'hiccups', in which matter alternates between being ejected by the propeller effect and being accreted onto the NS poles. Similar X-ray variability has subsequently been found in lightcurves from outbursts during the accreting phase of PSR J1023+0038 (Bogdanov et al. 2015), suggesting that this variability is somehow intrinsic to TMSPs as a class of objects.

1FGL J1227.9-4852 was first identified in the first Fermi/LAT source catalogue (Abdo et al. 2010). Hill et al. 2011 found that the $\gamma$-ray spectral characteristics of this source are consistent with known millisecond radio pulsars, although no radio pulsations were found. They suggested that this object could be associated with the X-ray source XSS J12270-4859. Before 2009, XSS J12270-4859 showed optical emission lines typical of an accretion disk (Pretorius 2009). Hill et al. 2011 suggested that XSS J12270-4859 may also be a TMSP, which switched from an accreting phase to a radio pulsar millisecond pulsar phase between 2009 and 2011. Subsequent studies have found pulsations in both the radio (Roy et al. 2015) and $\gamma$-ray (Johnson et al. 2015) emissions of this source, confirming the system contains a pulsar and establishing its spin period at $1.69 \mathrm{~ms}$.

XMM J174457-2850.3 is a neutron star X-ray binary. Although no X-ray or radio pulsations have been detected due to the faintness of the source, Degenaar et al. 2014a have found that the X-ray variability properties of this source are similar to those seen in other TMSPs. This object also exhibits extended low-luminosity states during outbursts, which Degenaar et al. 2014a suggest may be symptomatic of TMSPs.

3FGL J1544.6-1125 was also first identified in Fermi/LAT data. Bogdanov \& Halpern 2015 associated this object with the X-Ray source 1RXS J154439.4-112820. Due to the presence of $\gamma$-rays, as well as the presence of variability in the X-ray lightcurve similar to IGR J18245-2452, they proposed that this object is a TMSP in the accreting state. However, no pulsations from this system have been detected in the X-ray or the radio, so the pulsar period is not known. Bogdanov \& Halpern 2015 found a bimodality in count rate during the period of X-ray variability, suggesting that this behaviour can be explained as quick transitions between three quasi-stable accretion modes known as 'low' , 'high' and 'flaring'. This effect has also been seen in the TMSP IGR J18245-2452 (Ferrigno et al. 2014).

Strader et al. 2016 identified the $\gamma$-ray source, 3FGL J0427.9-6704, as a TMSP. They found that this source also displays X-ray variability similar to what is seen from the other known TMSPs. Finally, Rea et al. 2017 have proposed that the X-ray source XMM J083850.4-282759 may also be a TMSP. Although this source has not been detected in the gamma or the radio, the authors argued that X-ray variability coupled with $\mathrm{X}$-ray flaring seen from this object is reminiscent of similar behaviour seen in other TMSPs during subluminous disk states.

The phenomenology of currently known TMSPs is varied, and different methods have been used to conclude (or propose) that each individual system belongs to this class. The fact that 6 of the 7 objects show similar patterns of Xray variability during outburst suggests that this variability can be used as an indication that a system may be a TMSP. In this letter we present evidence that a $7^{\text {th }}$ object, the so-called 'Bursting Pulsar' GRO J1744-28, may also be associated with this family of objects.

\section{GRO J1744-28: THE BURSTING PULSAR}

GRO J1744-28, or the 'Bursting Pulsar' (hereafter BP, Paciesas et al. 1996), is an LMXB containing a $2.4 \mathrm{~Hz}$ pulsar and a $\sim 0.07-0.2 \mathrm{M}_{\odot}$ companion star (e.g. Sturner \& Dermer 1996; Finger et al. 1996b; Rappaport \& Joss 1997; Degenaar et al. 2014b); the orbital period of the systems is $11.8 \mathrm{~d}$ (Finger et al. 1996a; Sanna et al. 2017b). Estimates for the NS magnetic field range between 2 and $50 \times 10^{10} \mathrm{G}$ (Finger et al. 1996b; Degenaar et al. 2014b; D'Aì et al. 2015; Doroshenko et al. 2015). The BP is one of only two known systems that show 'Type II' X-ray bursts: bright, $\sim 10$ s-long non-thermonuclear X-ray flares. The "Rapid Burster" (i.e. MXB 1730-33; hereafter RB), is the other system (e.g. Lewin et al. 1976; Kouveliotou et al. 1996).

A number of physical models have been proposed to explain Type II bursts, including among others, viscous instabilities and interactions between the disk and the rotating NS magnetosphere (e.g. Taam \& Lin 1984; Spruit \& Taam 1993). None of the proposed models can fully reproduce the observed phenomenology (Lewin et al. 1993), nor explain the significant differences observed between the BP and the RP (e.g. Lewin et al. 1996, Court et al. in prep). Most importantly, so far it is not understood what differentiates the $\mathrm{RB}$ and the BP from the more than 100 known NS-LMXBs (e.g. Liu et al. 2007) which do not show Type II bursts. De Martino et al. 2013 previously noted similarities between Type II bursting and X-ray variability in the lightcurve of the TMSP 1FGL J1227.9-4852 (Hill et al. 2011). However, the authors note that the energetics are inconsistent with these phenomena being physically the same.

In Court et al. (in prep.), we performed a detailed analysis of all archival X-ray data (including RXTE, Swift, Chandra, XMM-Newton, Suzaku, NuStar, Fermi and INTE$G R A L)$ and found that the Type II phenomenology in the $\mathrm{BP}$ is much richer than previously thought (e.g. Giles et al. 1996): the characteristics of the flaring evolve with time and source luminosity. Near the end of this evolution, we observed periods of highly-sctructured and complex highamplitude X-ray variability. We refer to this variability as 'Structured Bursting', which is unlike what is seen other LMXBs but very similar to the 'hiccup' accretion observed in TMSPs.

\section{COMPARISON}

Rappaport \& Joss 1997 have previously suggested that the $\mathrm{BP}$ represents a slow X-ray pulsar nearing the end of its accreting phase. As such it is natural to compare this system with TMSPs, which are also believed to be systems approaching this evolutionary stage. In addition to this, 
Degenaar et al. 2014a have previously noted that the BP shows extended low-luminosity states during outburst, similar to those seen in the TMSP candidate XMM J1744572850.3 .

In Figure 1, we show RXTE lightcurves of 'Structured Bursting' from the BP alongside lightcurves from periods of 'hiccup' variability observed in the confirmed TMSPs PSR J1023+0038 and IGR J18245-2452. All three sources show similar patterns of X-ray variability: (i) Plateaus: periods of approximately constant count rate with high-amplitude flicker noise (all plateaus in a given observation have approximately the same mean rate), (ii) Dips: Periods of low count rate $(\lesssim 0.5$ of the rate in plateaus) with significantly less flicker noise, and (iii) Flares: Relatively short-lived increases of the count rate to values $\gtrsim 2$ times greater than the rate during plateaus. In TMSPs, these features are interpreted as representing three quasi-stable accretion modes: the 'high', 'low' and 'flaring' modes respectively (e.g. Bogdanov et al. 2015). The most significant difference is that, in general, the variability in the $\mathrm{BP}$ occurs on timescales $\sim 1$ order of magnitude longer than those in TMSPs.

In Figure 2 we show histograms of the 1 s-binned countrate from all RXTE observations of Structured Bursting in the 1996 (left) and 1997 (right) outbursts of the BP. As is the case for TMSPs, the histograms can be described with a number of log-Normally distributed populations: 3 populations in the 1996 outburst and 2 in the 1997 outburst. It is unclear why a population would be absent from the 1997 outburst, but some TMSPs have been observed to miss the 'high' mode during hiccup accretion (e.g. IGR J18245-2452, Ferrigno et al. 2014).

Detailed works on the low and high modes observed in the light curves of TMSPs show that X-ray pulsations are seen during both modes. Pulsations are fractionally weaker in the low state than the high state (for example varing between $4.0 \pm 0.2 \%$ and $16.8 \pm 0.2 \%$ in the TMSP IGR J182452452, Ferrigno et al. 2014). In the case of the BP, we detect pulsations both during the low and the high modes; much like in TMSPs, the pulsations are weaker in the low mode. For example in RXTE OBSID 10401-01-59-00 (in 1996), the pulsations had amplitudes of $3.5 \pm 0.2 \%$ and $4.9 \pm 0.2 \%$ respectively, while in OBSID 20078-01-23-00 (in 1997), the pulsations had amplitudes of $4.5 \pm 0.1 \%$ and $6.0 \pm 0.1 \%$ respectively. A reduction in pulse fraction in accreting pulsars has been interpreted as a change in accretion geometry due to a sudden decrease in the amount of matter reaching the compact object (e.g. Ibragimov \& Poutanen 2009), and as such this result provides direct evidence that the Structured Bursting in the BP is caused by switches between accretion and propeller-driven outflows.

TMSPs are amongst the only LMXBs which are also significant $\gamma$-ray sources (e.g. Hill et al. 2011). The Fermi point source 3FGL J1746.3-2851c is spatially coincident with the BP. While the field is too crowded to unambiguously associate 3FGL J1746.3-2851c with the BP, the existence of a $\gamma$-ray point source at this location is consistent with the possibility that the BP and TMSPs show the same phenomenology.

The spectral evolution of known TMSPs is varied. In PSR J1023+0038, the low, high and flaring modes all present similar spectra (Bogdanov et al. 2015). However in IGR J18245-2452, Ferrigno et al. 2014 have found a strong cor- relation between spectral hardness and intensity during hiccups, showing that there is spectral evolution over time in this source. In Figure 3 we show the hardness-intensity diagram of the BP during periods of Structured Bursting. We find a significant correlation, similar to what is seen in IGR J18245-2452 (Ferrigno et al. 2014). This is in contrast with other slow accreting pulsar systems such as Vela X-1, which show an anticorrelation between these parameters during periods of variability (Kreykenbohm et al. 2008).

\section{DISCUSSION}

In this letter we compare the lightcurve, spectral and timing properties of the Bursting Pulsar at the end of its 1996 and 1997 outburst with those observed from Transitional Millisecond Pulsars. The data suggest that the BP may have undergone "hiccup" accretion similar to that seen in TMSPs, during which transferred matter alternates between being accreted onto the poles of the NS and being ejected from the system by the 'propeller' effect (e.g. Ferrigno et al. 2014). This similarity raises the exciting prospect of studying the physics of TMSPs in a completely different regime.

Very recently Campana et al. 2017 proposed a universal relation between magnetic moment, spin frequency, stellar radius and luminosity at the boundary between accretion and the propeller effect. Any object that exists on one side of this boundary should be able to accrete, whereas objects on the other side should be in the propeller phase or not accreting at all. In Figure 4 we reproduce Campana et al. 2017's results and include our estimates for the BP during the periods of Structured Bursting. We find that the BP is consistent with lying on or near the boundary between propeller-mode and direct accretion, clustering with High Mass X-ray Binaries (as expected due to the BP's high magnetic field), and supporting the link between "hiccups" and Structured Bursting.

If the "hiccups" in the BP show that the system is transiting to a radio pulsar, then the BP should not lie in the $P-\dot{P}$ 'graveyard' region (e.g. van den Heuvel 1993). To our knowledge, there is no measurment yet of the NS spin down during the BP's X-ray quiescent state. Under the assumption that the $\mathrm{BP}$ becomes a radio pulsar, and that the possible spin down during that period is due to the same mechanism as those of the known radio pulsars, we can position the $\mathrm{BP}$ in the $P-\dot{P}$ diagram (the plot of pulsar spin $P$ against spin-down rate $\dot{P}$, not shown) by using the orbital period and estimates of its magnetic field. At $B \sim 2 \times 10^{11} \mathrm{G}$, the BP falls well outside of the pulsar graveyard. We note that Pandey-Pommier et al. (2014) and Russell et al. (2017) did not detect a significant radio source at the location of the BP during X-ray outburst. To our knowledge, there is no report of Radio detection/non-detection during X-ray quiescence.

In addition to the $\mathrm{BP}$, several additional sub- $10 \mathrm{~Hz}$ accreting X-ray pulsars have been discovered (e.g. GX $1+4$ and 4U 1626-67, Lewin et al. 1971; Rappaport et al. 1977). The reason behind the slow spins of these objects is poorly understood, but a number of these systems have been seen to undergo 'torque reversal' events, during which $\dot{P}$ switches sign (e.g. Chakrabarty et al. 1997a,b). In some sources, the magnitude of the spin-down during an event is of the same order magnitude as the preceding period of spin-up, resulting in little or no net spin change. Torque reversal events oc- 

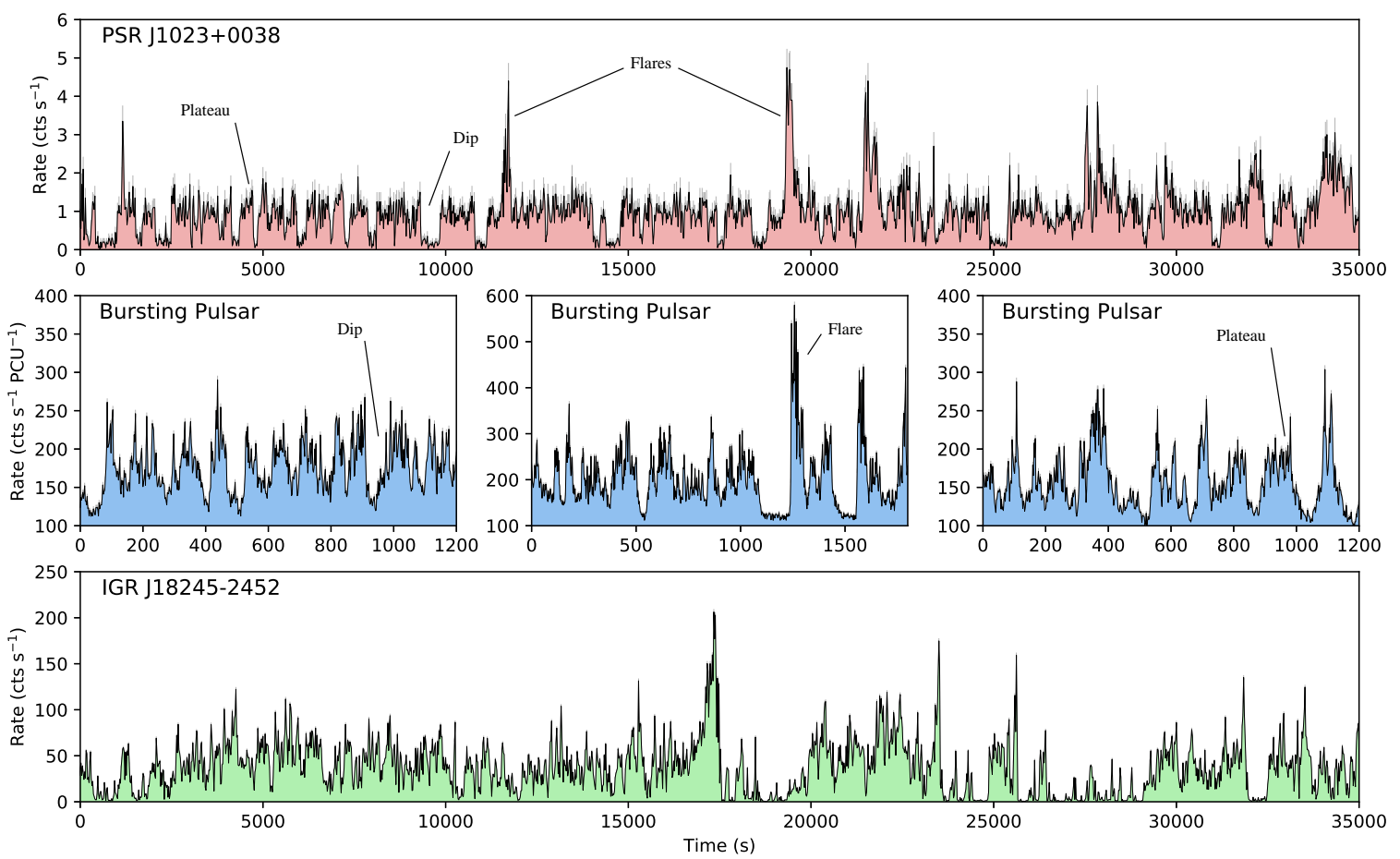

Figure 1. Top: $2-15 \mathrm{keV}$ XMM lightcurve from the TMSP PSR J1023+0038. Middle: $2-60 \mathrm{keV}$ RXTE lightcurves from the BP during its 1996 and 1997 outbursts, showing similar variability patterns to those seen in PSR J1023+0038. Bottom: 2-15 keV XMM lightcurve from the TMSP IGR J18245-2452. XMM lightcurves are shown from 2-15 keV so that they can be more directly compared with $R X T E$.
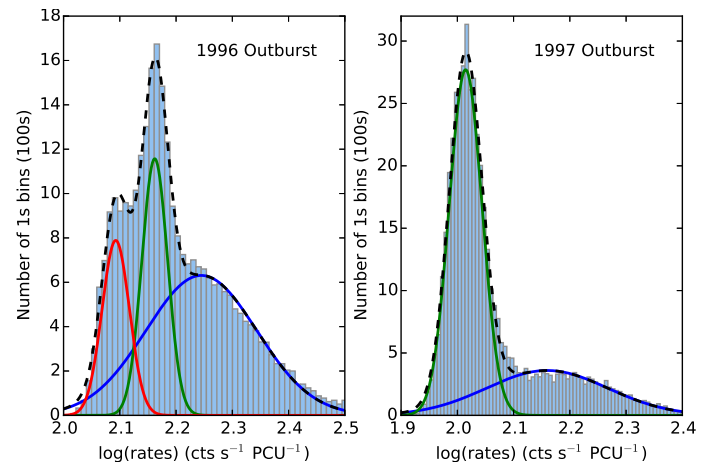

Figure 2. Histograms of the $1 \mathrm{~s}$ binned count rates from all RXTE observations of Structured Bursting in the 1996 (left) and 1997(right) outbursts of the BP. For the 1996 outburst, we fit the distribution with three Gaussians, while for the 1997 outburst we fit the distribution with 2 Gaussians. The individual Gaussians are plotted in solid lines, while the combined total is plotted in a dashed line.

cur irregularly, but the recurrence timescale varies between objects from weeks to decades (e.g. Bildsten et al. 1997).

Given that the BP has a strongly stripped stellar companion (Bildsten \& Brown 1997), a high magnetic field and shows significant spin-up during outburst (e.g. Finger et al. 1996b; Sanna et al. 2017b), it is difficult to explain its low spin by suggesting the system is young or that the angular momentum transfer is inefficient. Rappaport \& Joss 1997 suggest that the magnetic field and spin could be explained if much of the mass transfer in the system occurred before the primary became a neutron star, but they note that this scenario is inconsistent with the low mass of the donor star.

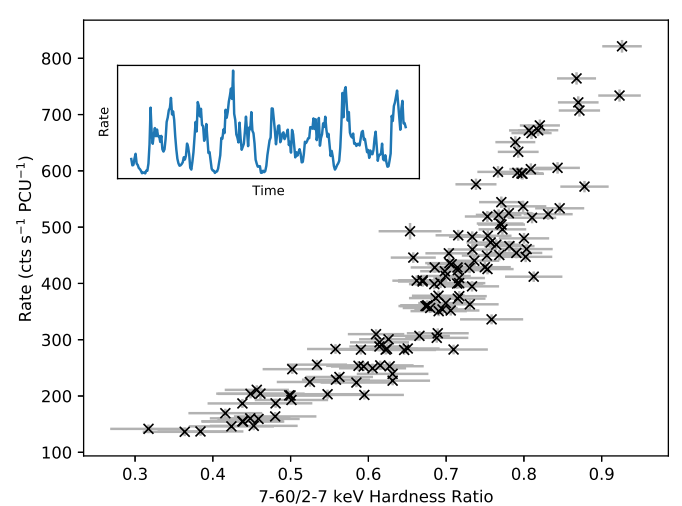

Figure 3. A 7-60/2-7 keV hardness-intensity diagram for $R X T E$ observation 10401-01-59-00; the lightcurve of this observation is shown in the inset. To correct for the high background of the region, we subtract the median count rate of $R X T E$ observation 30075-01-24-00 from each band; at this time, GRO J1744-28 was in quiescence. We find a strong correlation between hardness and count rate, with a Spearman Rank Correlation Coefficient of 0.93. Data for the hardness-intensity diagram are binned to $10 \mathrm{~s}$, while data for the lightcurve are binned to $5 \mathrm{~s}$.

Torque reversal events in the BP (similar to those seen in other slow accreting pulsars, e.g. Bildsten et al. 1997) could explain why the pulsar has failed to reach a spin rate on par with TMSPs. Although no torque reversal event has been reported from the BP, it is feasible that the recurrence timescale of such an event is longer than the $\sim 20$ years for which the object has been studied (this is consistent with the recurrence timescales seen in other slow accreting pulsars). 


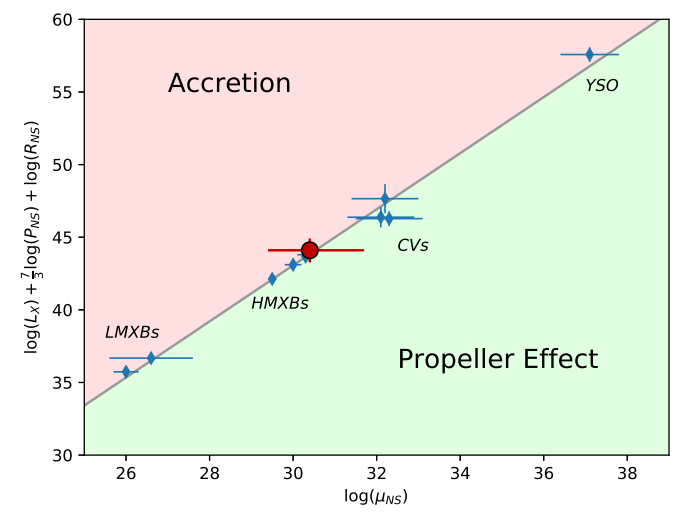

Figure 4. A plot of a number of objects ranging in scale from LMXBs and High-Mass X-ray Binaries (HMXBs) to Cataclysmic Variables (CVs) and Young Stellar Objects (YSOs) (blue diamonds). In each case, the object is plotted at the luminosity which defines its transition between propeller-mode accretion and free accretion. Campana et al. 2017 suggest that any object above the line of best fit accretes freely, whereas all objects below are in the propellor regime. The BP (red circle) is consistent with approaching this line during periods of Structured Bursting. Errorbars on the BP represent the range of the reported magnetic fields as well as a range of stellar radii between $10-20 \mathrm{~km}$. The range in luminosity for the BP is calculated using 1.5-25 keV RXTE/PCA flux, assuming a distance of between $4-8 \mathrm{kpc}$ (e.g. Kouveliotou et al. 1996; Gosling et al. 2007; Sanna et al. 2017b) and a bolometric correction factor of 1-3. Data on the other objects taken from Campana et al. 2017. $L$ is the bolometric luminosity of the object in $\operatorname{ergs~s}^{-1}, P$ is the period in $\mathrm{s}, R$ is the radius in $\mathrm{cm}$ and $\mu$ is the magnetic moment in Gauss $\mathrm{cm}^{3}$.

The discovery of torque reversal in the BP would strongly link it with the other known slow accreting pulsars.

The $\mathrm{BP}$ has a spin rate $\sim 2$ orders of magnitude less than previously known TMSPs, and a magnetic field $\sim 2$ orders of magnitude stronger, but it still shows lightcurve, timing and spectral behaviour which are remarkably similar to TMSPs. This raises the exciting prospect of exploring the physics of TMSPs in a previously unexplored physical regime. If the $\mathrm{BP}$ itself is a transitional pulsar, it should emit radio pulsations during $\mathrm{X}$-ray quiescence. Future detections of radio pulsations from this object would unambiguously confirm it as a transitional pulsar.

\section{REFERENCES}

Abdo A. A., et al., 2010, ApJS, 188, 405

Alpar M. A., Cheng A. F., Ruderman M. A., Shaham J., 1982, Nature, 300, 728

Altamirano D., Casella P., Patruno A., Wijnands R., van der Klis M., 2008, ApJ, 674, L45

Archibald A. M., et al., 2009, Science, 324, 1411

Backer D. C., Kulkarni S. R., Heiles C., Davis M. M., Goss W. M., 1982, Nature, 300, 615

Bildsten L., Brown E. F., 1997, ApJ, 477, 897

Bildsten L., et al., 1997, ApJS, 113, 367

Bogdanov S., Halpern J. P., 2015, ApJ, 803, L27

Bogdanov S., et al., 2015, ApJ, 806, 148

Campana S., Colpi M., Mereghetti S., Stella L., Tavani M., 1998, A\&ARv, 8, 279

Campana S., Stella L., Mereghetti S., de Martino D., 2017, preprint, (arXiv:1711.08233)

Chakrabarty D., et al., 1997a, ApJ, 474, 414
Chakrabarty D., et al., 1997b, ApJ, 481, L101

D'Aì A., et al., 2015, MNRAS, 449, 4288

De Martino D., et al., 2013, A\&A, 550, A89

Degenaar N., et al., 2014a, ApJ, 792, 109

Degenaar N., Miller J. M., Harrison F. A., Kennea J. A., Kouveliotou C., Younes G., 2014b, ApJ, 796, L9

Doroshenko R., Santangelo A., Doroshenko V., Suleimanov V., Piraino S., 2015, MNRAS, 452, 2490

Eckert D., et al., 2013, The Astronomer's Telegram, 4925

Ferrigno C., et al., 2014, A\&A, 567, A77

Finger M. H., Koh D. T., Nelson R. W., Prince T. A., Vaughan B. A., Wilson R. B., 1996a, Nature, 381, 291

Finger M. H., Wilson R. B., Harmon B. A., Hagedon K., Prince T. A., 1996b, IAU Circ., 6285

Giles A. B., Swank J. H., Jahoda K., Zhang W., Strohmayer T., Stark M. J., Morgan E. H., 1996, ApJ, 469, L25

Gosling A. J., Bandyopadhyay R. M., Miller-Jones J. C. A., Farrell S. A., 2007, MNRAS, 380, 1511

Hill A. B., et al., 2011, MNRAS, 415, 235

Ibragimov A., Poutanen J., 2009, MNRAS, 400, 492

Illarionov A. F., Sunyaev R. A., 1975, A\&A, 39, 185

Johnson T. J., et al., 2015, ApJ, 806, 91

Kouveliotou C., van Paradijs J., Fishman G. J., Briggs M. S., Kommers J., Harmon B. A., Meegan C. A., Lewin W. H. G., 1996, Nature, 379, 799

Kreykenbohm I., et al., 2008, A\&A, 492, 511

Lewin W. H. G., Ricker G. R., McClintock J. E., 1971, ApJ, 169, L17

Lewin W. H. G., et al., 1976, ApJ, 207, L95

Lewin W. H. G., van Paradijs J., van der Klis M., 1988, Space Sci. Rev., 46, 273

Lewin W. H. G., van Paradijs J., Taam R. E., 1993, Space Sci. Rev., 62, 223

Lewin W. H. G., Rutledge R. E., Kommers J. M., van Paradijs J., Kouveliotou C., 1996, ApJ, 462, L39

Liu Q. Z., van Paradijs J., van den Heuvel E. P. J., 2007, A\&A, 469, 807

Manchester R. N., Hobbs G. B., Teoh A., Hobbs M., 2005, AJ, 129, 1993

Paciesas W. S., Harmon B. A., Fishman G. J., Zhang S. N., Robinson C. R., 1996, IAU Circ., 6284

Pandey-Pommier M., Masetti N., Durouchoux P., 2014, The Astronomer's Telegram, 5904

Papitto A., et al., 2013a, Nature, 501, 517

Papitto A., et al., 2013b, The Astronomer's Telegram, 5069

Patruno A., Haskell B., Andersson N., 2017, ApJ, 850, 106

Pretorius M. L., 2009, MNRAS, 395, 386

Rappaport S., Joss P. C., 1997, ApJ, 486, 435

Rappaport S., Markert T., Li F. K., Clark G. W., Jernigan J. G., McClintock J. E., 1977, ApJ, 217, L29

Rea N., et al., 2017, MNRAS, 471, 2902

Roy J., et al., 2015, ApJ, 800, L12

Russell T., Degenaar N., Miller-Jones J., Tudor V., 2017, The Astronomer's Telegram, 10106

Sanna A., et al., 2017a, preprint, (arXiv:1711.03092)

Sanna A., et al., 2017b, MNRAS, 469, 2

Spruit H. C., Taam R. E., 1993, ApJ, 402, 593

Strader J., Li K.-L., Chomiuk L., Heinke C. O., Udalski A., Peacock M., Shishkovsky L., Tremou E., 2016, ApJ, 831, 89

Sturner S. J., Dermer C. D., 1996, ApJ, 465, L31

Szkody P., et al., 2003, AJ, 126, 1499

Taam R. E., Lin D. N. C., 1984, ApJ, 287, 761

Wijnands R., van der Klis M., 1998, Nature, 394, 344

van den Heuvel E. P. J., 1993, in Phillips J. A., Thorsett S. E., Kulkarni S. R., eds, Astronomical Society of the Pacific Conference Series Vol. 36, Planets Around Pulsars. pp 123-147 
This paper has been typeset from a $\mathrm{T}_{\mathrm{E}} \mathrm{X} / \mathrm{LAT}_{\mathrm{E}} \mathrm{X}$ file prepared by the author. 